\title{
Impact of HIV associated neurocognitive disorders on activities of daily living and its association with depression in outdoor patients undergoing HAART
}

\author{
Savita Saini", Kiran Vijay Barar \\ Department of Pharmacology, Sardar Patel Medical College, Bikaner, Rajasthan, INDIA.
}

\begin{abstract}
Objective: The prime objective was to determine the impact of HIV-associated neurocognitive disorders on activities of daily living and its association with depression. Material \& Methods: A prospective cohort study was conducted in ' 80 ' HIV positive patients, registered at ART Centre of PBM and AG Hospital, Bikaner. A test battery comprising of three scales was used i.e. International HIV Dementia Scale (IHDS), Activities of Daily Living(ADL) scale and PRIME-MD Patient Health Questionnaire(PHQ-9) to diagnose cognitive dysfunction, dependence in $A D L$ and depression respectively. Results: The $32.50 \%(n=26)$ of the patients were diagnosed to have cognitive dysfunction (IHDS Score $<10$ ) while remaining $67.50 \%$ $(n=54)$ of the patients were normal (IHDS Score $>10)$. Out of these cognitively impaired patients, $n=2$ patients were found to be dependent in $>2 A D L$ while remaining patients were able to perform their $A D L$ independently. No significant association was found between depression and cognitive dysfunction. Conclusion: Patients having score of $<10$ on IHDS were further classified on the basis of dependence in ADL. The patients having high degree of depressive symptoms were more likely to have cognitive dysfunction but the results were not significant.
\end{abstract}

Key words: HIV-associated neurocognitive disorder, Activities of daily living (ADL) scale, Patient health questionnaire (PHQ), International HIV dementia scale (IHDS), Asymptomatic neurocognitive impairment, Mild neu- rocognitive disorder, HIV associated dementia.

PICTORIAL ABSTRACT
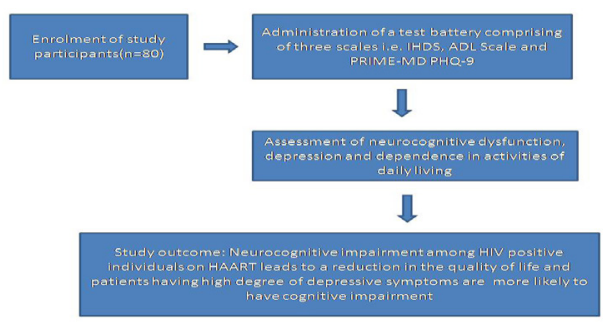

Correspondence :

Dr. Savita Saini,

Professor and Head, Assistant Professor, Department of Pharmacology, C-21, Gyansarovar colony, Kota, Rajasthan, INDIA

Phone no: 9460680004

E-mail: drsavitasaini04@gmail.com

DOI: 10.5530/jyp.2016.3.19

\section{INTRODUCTION}

The HIV infection has been declared as one of the worst pandemic, which is often associated with neurocognitive and psychological impairment. The neurocognitive complications associated with HIV have recently been characterized as HIV-associated neurocognitive disorders (HAND). HAND defines three categories of dysfunction: asymptomatic neurocognitive impairment (ANI), mild neurocognitive disorder (MND) and HIV-associated dementia (HAD). ${ }^{1}$ Since the introduction of highly active antiretroviral therapy (HAART), the incidence of severe forms of HAND has declined significantly, whereas the prevalence of the milder forms has increased. ${ }^{2,3}$

There is clinical significance of identifying the milder forms of HAND, as they can have a significant impact on the lives of people living with HIV. They have been shown to interfere with medication adherence, workplace performance, driving and ability to carry out tasks independently. ${ }^{4,5,6}$

Major depressive disorder (MDD) is the most common neuropsychiatric symptom associated with HIV. MDD may share similar pathological features (e.g. frontostriatal circuits) to HIV infection and has been independently associated with subtle cognitive decline in areas including attention, psychomotor speed, learning and memory, and executive functioning. Despite the similar underlying neural abnormalities, most studies have failed to find synergistic effects of depression and HIV infection on cognition. ${ }^{7}$
The neuropsychological impairment among HIV-positive patients remains largely unrecognized because it is not routinely screened for, despite this recommendation in HIV treatment guidelines. Early and periodical neuropsychological screening of HIV positive asymptomatic individuals is a must in future to spot the neurocognitive deficits at an early stage. ${ }^{8}$

\section{MATERIAL AND METHODS}

\section{Study design}

This was a prospective cohort study undertaken between Oct.2011 and Sept.2012 in ' 80 ' HIV/AIDS patients undergoing antiretroviral treatment. All the participants were registered with the ART Centre of PBM and AG Hospital, Bikaner, Rajasthan, India.

\section{Ethical considerations}

The study obtained ethical approval from the Ethical Committee of Sardar Patel Medical College, Bikaner. Study participants were invited to consent after being provided with adequate information about the study. To be eligible for inclusion in this study, following criteria were used for the participants-

- HIV positive patients on stabilized HAART for more than 6 weeks

- Men/women between the age 21-50 years

- Ambulatory patients with CD4 count above 200 cells $/ \mathrm{mm}^{3}$

- Ability to comprehend study procedures 
Seriously ill patients, pregnant women, patients having severe psychiatric disorder or any substance abuse were excluded from the study.

\section{Data collection tools}

All the participants underwent neurocognitive examination, assessment of depression and activities of daily living. The tools included a sociodemographic questionnaire, the International HIV Dementia Scale (IHDS), Activities of Daily Living (ADL) scale and PRIME-MD Patient Health Questionnaire (PHQ-9).

According to modified updated American Academy of Neurology (AAN) criteria, patients with HAND are classified on the basis of neurocognitive impairment and severity of dependence in activities of daily living (ADL). To assess neurocognitive impairment we used IHDS while dependence in $\mathrm{ADL}$ was assessed by ADL scale.

\section{International HIV Dementia Scale}

The IHDS is a screening measure of neurocognitive impairment that assesses for memory impairment, motor and psychomotor speed. It can be used in a clinic setting, does not require one to be proficient in English and is brief and inexpensive. Here a cut-off score of $\leq 10$ was taken to screen dementia cases. ${ }^{9}$

\section{Activities of daily living scale}

This questionnaire was selected for its wide use and demonstrated validity in studies of medically ill and dementia population, including HIV. It includes 2 scales namely Katz basic activities of daily living scale and Lawton-Brody Instrumental activities of daily living scale. ${ }^{10,11}$ It is a '14-item scale' that measures physical self-maintenance activities and instrumental activities of daily living. Patients were classified as 'ADL dependent' if they reported decline in two or more ADL.

\section{PRIME-MD Patient Health Questionnaire (PHQ-9) for endogenous depression}

The PHQ-9 follows the Diagnostic and Statistical Manual-IV (DSM-IV) Criteria for screening patients for current depression. If 5 or more of the 9 symptoms are present and one of the symptoms is sadness/ hopelessness or anhedonia, then a diagnosis of major depressive disorder is supported..$^{12,13}$

\section{Statistical Analysis}

Means and Standard Deviations (SD) were calculated for continuous variables. To analyse the association between the various factors and cognitive dysfunction, chi square test was employed. The $p$ value of less

Table 1: Socio-demographic characteristics of participants ( $N=\mathbf{8 0})$

\begin{tabular}{cc}
\hline Parameter & Number of participants (\%) \\
\hline Age (in years) & $13(16)$ \\
$21-30$ & $43(54)$ \\
$31-40$ & $24(30)$ \\
$41-50$ & \\
Gender & $45(56)$ \\
Male & $35(44)$ \\
Female & \\
Marital status & $58(73)$ \\
Married & $4(5)$ \\
Single & $18(22)$ \\
Widow/Divorced & \\
Educational status & $41(51)$ \\
Illiterate & $25(31)$ \\
Primary school & $10(13)$ \\
Secondary school & $4(5)$ \\
College \& above &
\end{tabular}

Table 2: Classification of HIV patients based on neurocognitive tests

\begin{tabular}{cc}
\hline Nature of neurocognitive dysfunction & No. of patients (\%) \\
\hline HIV-Associated Dementia (HAD) & Nil \\
Mild Neurocognitive Disorder (MND) & $2(2.5)$ \\
Asymptomatic Neurocognitive Impairment (ANI) & $24(30)$ \\
No cognitive impairment & $54(67.5)$ \\
\hline
\end{tabular}

Table 3: Depressive symptom and cognitive dysfunction

\begin{tabular}{ccccc}
\hline Depressive symptom & Cognitive dysfunction & $\begin{array}{c}\text { No cognitive } \\
\text { dysfunction }\end{array}$ & $\chi^{2}$ & p value \\
\hline Minimal (1-4) & $17(65.38)$ & $40(74.07)$ & 0.009 & $>0.05$ \\
Mild (5-9) & $5(19.23)$ & $10(18.52)$ & 0.628 & $>0.05$ \\
Moderate (10-14) & $3(11.54)$ & $4(7.41)$ & 0.375 & $>0.05$ \\
Moderately severe (15-19) & $1(3.84)$ & Nil & - & - \\
Severe (20-27) & Nil & Nil & - & - \\
\hline
\end{tabular}




\section{$\square \leq 10 \quad \square>10$}

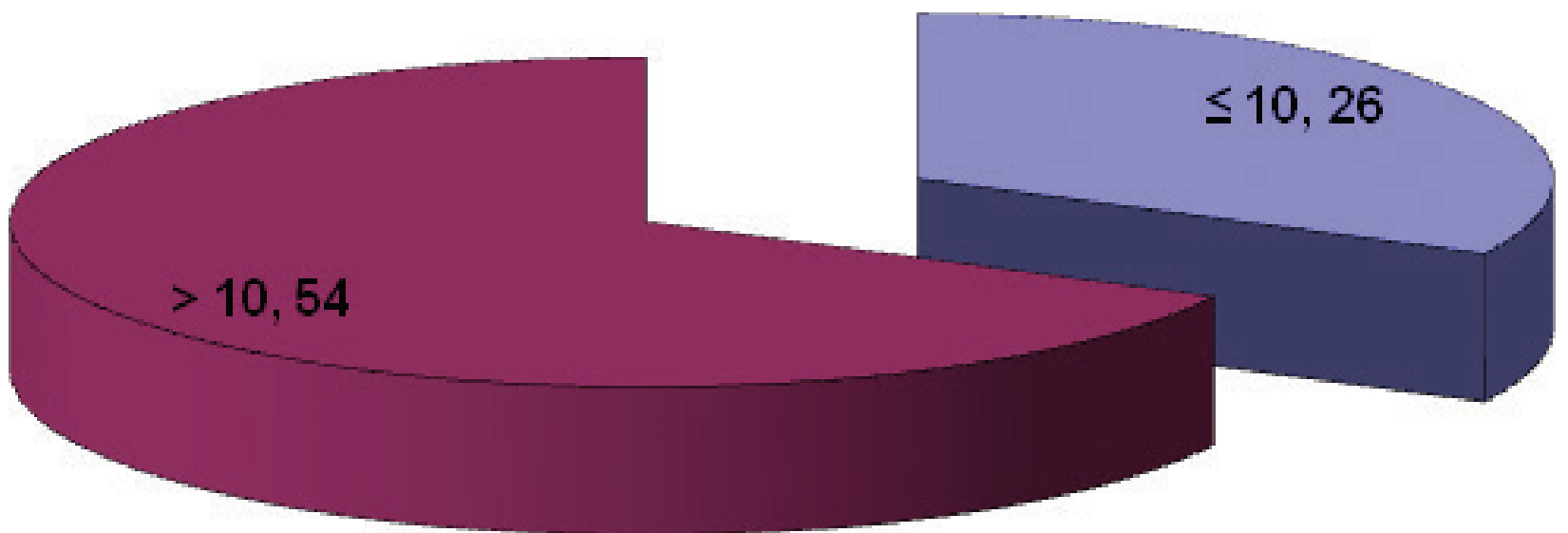

Figure 1: Distribution of patients according to IHDS score.

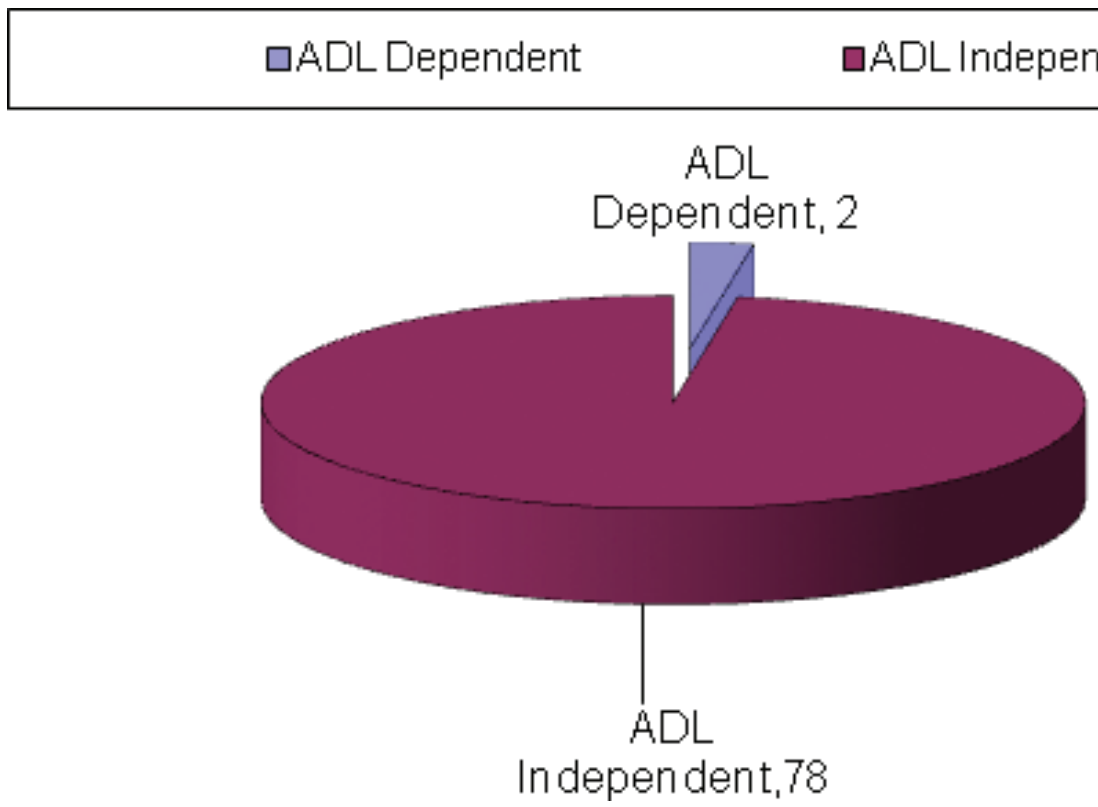

Figure 2: Distribution of Patients according to ADL score.

than 0.05 was considered as statistically significant. All statistical analysis was done by using 'INDOSTAT software'.

\section{RESULTS}

Of the ' 80 ' study participants, 45 were males (56\%) and 35 were females (44\%). The socio-demographic characteristics of the participants are presented in Table 1.

In present study, using a cut-off score of 10 or less on the IHDS, patients were classified as having cognitive dysfunction and normal cognitive function. Out of total ' 80 ' HIV-positive patients, 26 patients (32.50\%) had scored $\leq 10$ on IHDS while remaining 54 patients $(67.50 \%)$ scored $>10$ (See Figure 1).
Further classification of cognitively impaired patients was done on the basis of dependence in ADL. The $2.50 \%(\mathrm{n}=2)$ of the patients were found to be dependent in $\geq 2$ ADL while the $97.50 \%$ ( $n=78)$ of the patients were able to perform their ADL independently i.e. ADL independent (See Figure 2).

\section{Classification of HAND according to modified updated American Academy of Neurology (AAN) criteria}

Patients who were found to be dependent in ADL and had scored $<10$ on the IHDS were classified under mild neurocognitive disorder (MND) category of HAND whereas the patients who were ADL independent and had a score of $<10$ on IHDS were categorised under asymptomatic 
neurocognitive impairment (ANI) category. The most severe form of HAND i.e. HAD was not observed in any of the subjects (See Table 2).

\section{PRIME-MD PHQ-9 scoring for endogenous depression}

The patients having high degree of depressive symptoms were more likely to have cognitive dysfunction but the results were not significant ( $p>0.05)$ (See Table 3).

\section{DISCUSSION}

In today's world, HIV/AIDS has poorly affected the vast majority of population and it still remains the major health burden across the world. With the prevalence of milder forms of HAND increasing and limited resources available for formal neuropsychological examinations, there is a critical need to be able to screen people with neurocognitive disorders. Asymptomatic Neurocognitive Impairment (ANI) is very common in AIDS patients and it was observed in $30 \%$ of our study subjects. All of these subjects were 'ADL independent'. This finding is an agreement of finding of Lawler $\mathrm{K}$ et al, in which majority of the patients having cognitive dysfunction were asymptomatic. ${ }^{14}$

HIV-associated Mild Neurocognitive Disorder (MND) constituted 2.5\% of our study population who were found to be 'ADL dependent'. Similar finding was observed in a previous study by Muniyandi K et al. ${ }^{15}$ None of the patients had HAD which was in concordance with two prospective studies from India which also reported low incidence of HAD (1 to $2 \%)^{16,17}$
One of the factors discussed in relation to cognitive dysfunction in HIV positive individuals is co-morbid depression. In concordance with previous studies ${ }^{18,7}$ the present study also did not observed significant association between depression and cognitive dysfunction.

\section{CONCLUSION}

To conclude, the neuropsychological impairment among HIV positive patients on ARV therapy leads to a reduction in the quality of life and the patients having high degree of depressive symptoms are more likely to have cognitive dysfunction. It is an important challenge due to the high prevalence of HAND and its concomitant consequences in relation to morbidity and mortality. The presence of mild neurocognitive disorder in HIV is predictive of HAD, the most severe form of HAND. Therefore, early and periodical neuropsychological screening of HIV positive individuals is a must in future, to spot the neurocognitive deficits at an early stage.

\section{ACKNOWLEDGEMENT}

The authors are thankful to the entire faculty of the ART Center and Medicine Department, PBM and AG Hospital, Bikaner, Rajasthan (India), for their cooperation and support during this study.

\section{CONFLICT OF INTEREST}

The author have no conflict of interest to declare.

\section{ABBREVIATIONS USED}

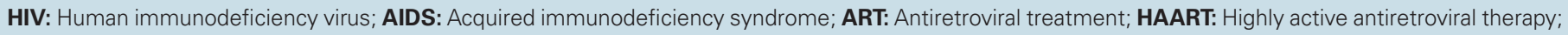

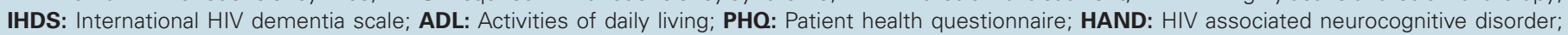
ANI: Asymptomatic neurocognitive impairment; MND: Mild neurocognitive disorder; HAD: HIV associated dementia; MDD: Major depressive disorder; AAN: American academy of neurology; DSM: Diagnostic and statistical manual; SD: Standard deviation.

\section{ABOUT AUTHORS}

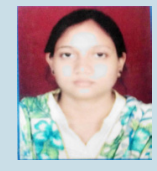

Dr. Savita Saini: Is Assistant professor at the department of Pharmacology, Sardar Patel Medical College, Bikaner, Rajasthan (India). She has completed MD (Pharmacology) in 2013 at S.P.Medical college, Bikaner. She has nine research publications.

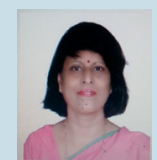

Dr. Kiran Vijay Barar: Is Senior Professor at the Department of Pharmacology, S.P.Medical College, Bikaner, Rajasthan, India. She is also serving as Honorary Professor at "Albert Schiwtzer International University" (ASIU), Geneva, Switzerland since yr 2000. She has published about "75" papers and has been an Editor, Reviewer and Writer/Columnist. She has been a Meritorious (Gold Medalist) throughout, Winner of various "Distinctions, Awards and Prizes" during her whole academic career and she has been recipient of National Merit Scholarship for four years (1976-1980). She has served as Resource-Person/Moderator in various teaching programs/CME/group-discussions and also Deliberated "Lead-Lectures" in Neurodegenerative/Alzheimer"s Disease(s), Biopharmaceuticals/ Biosimilars, Monoclonal-Antibodies etc. Recently, on Drug Regulatory Affairs and Obesity, Dr.Kiran deliberated lectures at "Singapore" and "Las Vegas" as Invited Speaker, Which have been published in Obesity journal in 2012-2014.

\section{REFERENCES}

1. Antinori A, Arendt G, Becker JT. Updated research nosology for HIV-associated neurocognitive disorders. Neurology. 2007;69(18):1789-99.

2. Heaton RK, Clifford DB, Franklin DR Jr, Woods SP, Ake C, Vaida F. HIV-associated neurocognitive disorders persist in the era of potent antiretroviral therapy: CHARTER Study. Neurology. 2010;75(23):2087-96.

3. Albert SM, Weber CM, Todak G, Polanco C, Coulse R, McElhiney M. An observed performance test of medication management ability in HIV: relation to neuropsychological status and medication adherence outcomes. AIDS Behav. 1999;3(2):121-8.

4. Heaton RK, Velin RA, McCutchan JA, Gulevich SJ, Atkinson JH, Wallace MR Neuropsychological impairment in human immunodeficiency virus-infection: implications for employment. HNRC Group. HIV Neurobehavioral Research Center. Psychosom Med. 1994;56(1):8-17.
5. Marcotte TD, Wolfson T, Rosenthal TJ, Heaton RK, Gonzalez R, Ellis RJ. A multimodal assessment of driving performance in HIV infection. Neurology. 2004;63(8):1417-22.

6. Woods SP, Iudicello JE, Moran LM, Carey CL, Dawson MS, Grant I. HIV-associated prospective memory impairment increases risk of dependence in everyday functioning. Neuropsychology. 2008;22(1):110-7.

7. Cysique LA, Deutsch R, Atkinson JH, Young C, Marcotte TD, Dawson L. The HNRC Group. Incident major depression does not affect neuropsychological functioning in HIV-infected men. J Int Neuropsychol Soc. 2007;13(1):1-11.

8. Chandler G, Himelhoch S, Moore RD. Substance Abuse and Psychiatric Disorders in HIV-positive patients: epidemiology and impact on antiretroviral therapy. Drugs. 2006;66(6):769-9. 
9. Sacktor NC, Wong M, Nakasujja N, Skolasky RL, Selnes OA. The International HIV Dementia Scale: a new rapid screening test for HIV dementia. AIDS. 2005;19(13):1367-74.

10. Shelkey M, Wallace M. Katz Index of Independence in Activities of Daily Living (ADL). Director. 2000;8(2):72-3.

11. Lawton MP, Brody EM. "Assessment of older people: self-maintaining and instrumental activities of daily living". Gerontologist. 1969;3:179-86.

12. Prime -MD Patient Health Questionnaire ( $\mathrm{PHQ}-9$ ) available at www.headstartmt.org/Health /PHQ-9.pdf.

13. American Psychiatric Association: Diagnostic and Statistical Manual of Mental Disorders, Fourth Edition (DSM-IV) 1994.

14. Lawler K, Mosepele M, Ratcliffe S. Neurocognitive impairment among HIV positive individuals in Botswana: a pilot study. Journal of the international AIDS Society. 2010;13(1):15, Pg 01-09.

15. Muniyandi K, Venkatesan J, Arutselvi T, Jayaseelan V. Study to assess the prevalence, nature and extent of cognitive impairment in people living with AIDS Indian J Psychiatry. 2012;54(2):149-53.

16. Satishchandra P, Nalini A. Profile of neurologic disorders associated with HIV/AIDS from Bangalore, south India. Indian J Med Res. 2000;111 (issue missing):14-23.

17. Wadia RS, Pujari SN. Neurological manifestations of HIV disease. J Assoc Physicians India. 2001;49:343-8.

18. Chan LG, Kandiah N, Chua A. HIV-associated neurocognitive disorders (HAND) in a South Asian population-contextual application of the 2007 criteria. BMJ Open. 2012;2(1):e000662. 